\title{
Online Policies for Opportunistic Virtual MISO Routing in Wireless Ad Hoc Networks
}

\author{
Cristiano Tapparello, Stefano Tomasin and Michele Rossi \\ Department of Information Engineering, University of Padova, \\ via Gradenigo 6/B - 35131, Padova, Italy (firstname.lastname@ dei.unipd.it)
}

\begin{abstract}
Cooperative routing has been shown to be an effective technique to improve the throughput/delay performance of multi-hop wireless ad hoc networks. In addition, suitable cooperation selection policies also allow for a reduction of the overall energy expenditure. In a previous study, we proposed a centralized algorithm to obtain optimal cooperation selection policies in multi-hop networks with the aim of minimizing a linear combination of energy and delay costs. In this paper, we look at this problem from a different angle, devising three online and fully distributed algorithms which only exploit local interactions for the selection of the cooperators. The first technique selects at each hop a fixed number of nodes having the minimum distance with respect to the destination. The second one adopts a look-ahead strategy, which selects a fixed number of nodes at each hop, according to their expected advancement toward the destination. The third technique utilizes a more refined look-ahead strategy, which dynamically adjusts the number of nodes that cooperate at each hop. Numerical results are thus presented for the proposed techniques, comparing them against the optimal centralized strategy and competing algorithms from the literature. These results indicate that our techniques improve upon existing distributed approaches and achieve close-to-optimal performance.
\end{abstract}

\section{INTRODUCTION AND RELATED WORK}

Cooperation for the transmission in wireless networks has been proposed as an effective way of increasing the throughput and also potentially reducing energy consumption. Early studies dealt with two-hop transmissions [1], where the communication between two nodes is assisted by a third node, usually located within them. As an example, [2] presents a distributed routing protocol that at each hop selects the best relay node based on instantaneous channel measurements. In this paper we focus on multiple hop transmissions where cooperative routing becomes relevant. Various approaches have been investigated and the paradigms of virtual antenna arrays or cooperative diversity have been proposed. In [3], [4] and [5] it is proposed that a subset of nodes that have received the information at a given hop cooperate in forwarding it to nodes placed farther away. However, the routing path is calculated ignoring cooperation. In [6] the number of cooperative nodes is computed during the network initialization phase and kept constant throughout the entire end-to-end path. Although suboptimal, these approaches improve the throughput and reduce energy consumption.

With opportunistic routing, decisions are made in an online manner by choosing the relay at each hop based on the actual transmission outcomes as well as a rank ordering of neighboring nodes. For this approach, it has been shown that the impact of poor wireless links can be mitigated by exploiting the broadcast nature of wireless transmissions, also referred to as wireless broadcast advantage (WBA), [7]. Without considering cooperative diversity, the superiority of opportunistic routing when compared to traditional routing has been provided through a Markov decision theoretic formulation in [8], while a distributed algorithm for optimal policies is presented in [9].

We derived in [10] the optimal cooperator selection policies exploiting opportunistic routing and we provided an analytical tool meant for centralized and off-line use. Distributed protocols combining opportunistic routing with cooperative diversity in virtual multiple input single output (MISO) transmissions and space-time block codes have been proposed in [11] and [12]. While these protocols exploit opportunistic routing for the selection of the relay nodes, the end-to-end path is still calculated ignoring cooperation.

This paper extends the work in the literature, and in particular [10]. We consider a multihop wireless network where a source node sends a message to a destination node and intermediate nodes that decode this message forward it to the next hop until the destination is reached. At each hop, relay nodes (referred to as cooperators) implement a virtual antenna array and realize a distributed space-time coding scheme with decode and forward (DF). The focus of this paper is on devising efficient online and localized cooperator selection policies. In particular, cooperating nodes are selected on the basis of a) their knowledge of the local topology and b) the fact that they correctly decoded the message at the previous hop. In [10] a centralized solution to this problem has been proposed, where full knowledge of the topology is exploited. In this paper, we propose three techniques that aim at solving the problem in a distributed fashion. The first technique selects at each hop a fixed number of nodes having the minimum distance with respect to the destination. The second one performs a look-ahead strategy, which selects a fixed number of nodes according to their expected advancement toward the destination. The third technique dynamically adjusts the number of cooperating nodes at each hop, thus exploiting a further degree of freedom in the local optimization process. We compare our online cooperator selection schemes against the optimal centralized approach of [10], showing that they attain close-to-optimal results. In addition, we show the superiority 
of our techniques with respect to the heuristic protocol of [12], which already outperforms other existing solutions.

The rest of the paper is organized as follows. In Section II we present the system model and the cooperator selection problem. In Section III we present our online algorithms for cooperative routing. In Section IV we prove the effectiveness of the proposed algorithms by comparing them against optimal policies and competing online heuristics from the literature. Finally, Section V concludes the paper.

\section{SySTEM MODEL}

Consider a wireless network consisting of a set $\mathcal{N}$ of nodes spread out according to any distribution. Time is slotted with a slot corresponding to the fixed transmission time of a packet and all nodes are synchronized at the slot level. We want to deliver a message from a source node $s$ to a termination node $t$.

In the first transmission slot, only the source node $s$ has the message and broadcasts it to all the nodes in the network. After this transmission, a set $\mathcal{R}_{1} \subseteq \mathcal{N}$ of nodes has correctly decoded the message and, according to the DF scheme, they are all eligible for transmitting it in the next hop. However, only a subset $a_{2} \subseteq \mathcal{R}_{1}$ will actually cooperate in the second time slot, and they do so by simultaneously transmitting the message with a distributed space-time code. Decoding and cooperative retransmission are iterated until the termination node $t$ is reached. At the generic hop $i, i=1,2, \ldots$, nodes in set $a_{i}$ simultaneously broadcast the message, and they are chosen from the set $\mathcal{R}_{i-1}$ of nodes that have correctly decoded the message in the previous time slot. $a_{i}$ is referred to as the relay node set in slot $i$.

\section{A. Link Model and Packet Outage Probabilities}

Each node is equipped with one antenna, and when the nodes in a set $a \subseteq \mathcal{N}$ cooperatively transmit, the total number of transmit antennas is $|a|$. We assume that nodes operate in half-duplex mode and that the same power is used at all transmit antennas. Furthermore, we assume no instant channel knowledge at the transmitter, i.e., transmit nodes are not aware of channel conditions of surrounding nodes.

As transmit nodes are not aware of channel conditions, messages are encoded with a capacity-achieving code having spectral efficiency $R$. When the channel capacity, normalized with respect to the bandwidth, is below $R$, outage occurs. In this case the packet is not decoded at the receiving node and is discarded. Let $C(a, n)$ be the capacity of the channel between nodes in $a$ and node $n$, normalized with respect to the bandwidth. Then, the outage probability is

$$
p_{\text {out }}(a, n)=\mathrm{P}[C(a, n)<R] \text {. }
$$

The computation of $p_{\text {out }}(a, n)$ has been detailed in [10] in a scenario with path loss and fading.

\section{B. Cooperator Selection Objectives}

The evolution of the considered cooperative multihop network can be described by a Markov chain, where a generic state $x$ of the chain represents the set of nodes that have correctly decoded the message in a particular time slot, [10]. For each generic state $x$ for which $t \notin x$, let $a \subseteq x$ be the set of nodes that will cooperate in the next slot. The dynamics of the network are captured by transition probabilities $p_{x y}(a)$, describing the probability that nodes in state $y$ correctly decode the message after it has been transmitted by nodes in the set $a$, when the state was $x$. In addition, a positive normalized cost is associated with each transition

$$
c(x, y, a)=\alpha C_{E}(x, a, y)+(1-\alpha) C_{D}(x, a, y),
$$

where $C_{E}(x, a, y)=|a|$ (energy cost) accounts for the energy spent in transmitting the message and $C_{D}(x, a, y)=1$ (delay cost) accounts for the delay, in number of hops, associated with a path from $s$ to $t$, while $\alpha \in[0,1]$ is a parameter that we tune to drive the optimization. Note that the cost is normalized with respect to the cost associated to a single packet transmission.

The objective of our optimization is the minimization of the average cost over all possible paths in the network, according to a routing policy. In particular, for each possible state $x$ we have to identify an action $a^{*}(x)$ so that the message reaches the termination state, where the destination $t$ has correctly decoded the message, following the path with minimum average cost. To this end, [10] presents an analytical tool that returns the optimal policies for general network topologies. The performance of these policies represent an upper bound on the performance achievable by any routing algorithm.

\section{Heuristic Routing Policies}

The computation of the optimal policies, according to the objectives introduced in the previous section, requires a complete knowledge of the network topology and an offline, centralized solver [10]. Here, we present three heuristic policies suitable for a distributed implementation and having a lower computational complexity.

\section{A. K-Closest}

The idea of the $K$-Closest policy is to have a fixed number of relays retransmitting the message at each time slot and select them according to their distance to the termination node.

For any node $n \in \mathcal{N}$ let $\delta_{n}=d_{n, t}$ be the distance between node $n$ and the termination node $t$. We assume that each node $n$ can collect this proximity metric from all nodes closer to the destination than itself. All the nodes that correctly receive the message in time slot $i$ are ranked by the transmitters according to $\delta_{n}=d_{n, t}$, i.e., the ordered set is

$$
\mathcal{R}_{i}=\left\{r_{i}^{(1)}, r_{i}^{(2)}, \ldots, r_{i}^{\left(\left|\mathcal{R}_{i}\right|\right)}\right\},
$$

with

$$
\delta_{r_{i}^{(k)}} \leq \delta_{r_{i}^{(k+1)}}, \quad k=1,2, \ldots,\left|\mathcal{R}_{i}\right|-1 .
$$

With the $K$-Closest policy at most $K$ nodes cooperate in each time slot, and they are selected among those closest to the 


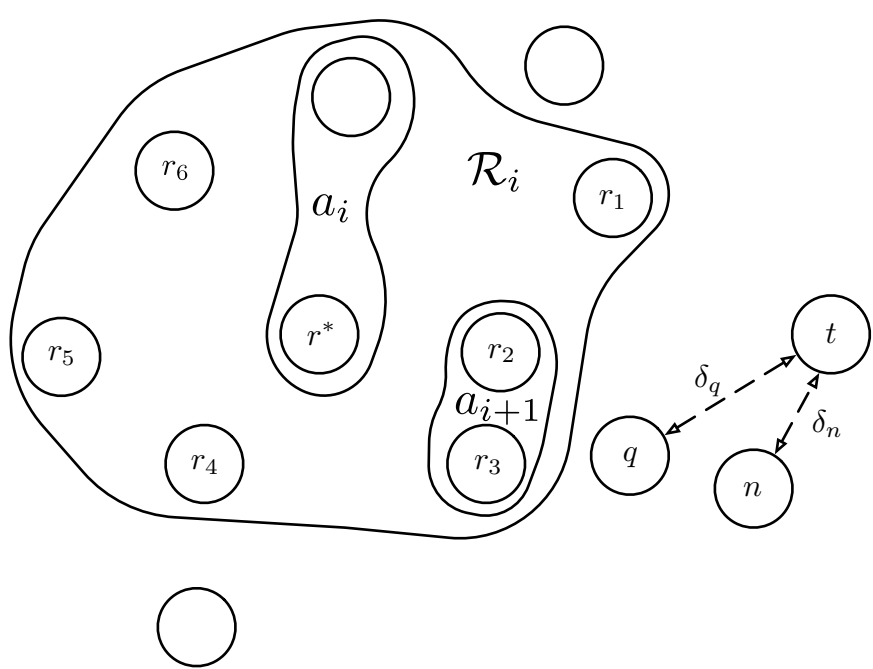

Fig. 1. Example of a scenario where $K$-Closest would choose an unreliable relay set.

destination. Thus, the set of nodes that cooperatively transmit at slot $i+1$ is

$$
a_{i+1}=\left\{r_{i}^{(1)}, r_{i}^{(2)}, \ldots, r_{i}^{\left(\min \left\{K,\left|\mathcal{R}_{i}\right|\right\}\right)}\right\} .
$$

Note that if less than $K$ nodes correctly decoded the message at slot $i$, they are all elected as relays in the next time slot $i+1$.

\section{B. K-One Step Look Ahead (K-OSLA)}

The $K$-Closest policy exploits the knowledge of the geographical distance between each potential relay and the termination node $t$. However, due to the limited amount of information that it uses, $K$-Closest has the potential drawback of choosing relays having a small number of neighbors in their proximity. Notably, this may increase the average number of retransmissions necessary to reach the next set of relays. As an example, consider the scenario of Fig. 1 where the number of nodes that cooperate for the transmission in each time slot is $K=2$. At a generic time slot $i$, nodes in the relay set $a_{i}$ cooperatively transmit the message and nodes in set $\mathcal{R}_{i}$ correctly decode it. Following the rationale of $K$-Closet, nodes $r_{1}$ and $r_{2}$ would be selected as the relays for the next transmission slot $i+1$, since they are the closest nodes to the destination $t$. However, despite the fact that node $r_{1}$ is the closest to $t$, it does not have additional intermediate nodes between itself and $t$. Additionally, as $r_{1}$ is quite distant from the destination, it will give a small contribution into the successful forwarding of the packet towards it. Choosing nodes $r_{2}$ and $r_{3}$ as the next relay set $a_{i+1}$ will instead avoid unnecessary retransmissions by taking advantage of multihopping through the neighboring nodes $q$ and $n$. In what follows, we extend the $K$-Closest heuristic with a suitable metric, which keeps into account the presence of neighboring nodes in order to avoid the discussed drawback.

Formally, let $q \in a_{i}$ be a node that transmits the message at slot $i$ and $n$ be a candidate node for the (cooperative) transmission of the message in the next time slot $i+1$. The difference between the distances of node $q$ and node $n$ with respect to the termination node is denoted as

$$
g_{q, n}=\delta_{q}-\delta_{n},
$$

which describes the geographical advancement toward $t$ provided by $n$. For each node $q \in \mathcal{N}$ we can also compute the expected maximum advancement toward $t$ provided only by node $q$ without the support of other cooperating nodes, i.e.

$$
g_{q}=\sum_{n: \delta_{n} \leq \delta_{q}} g_{q, n}\left[1-p_{\text {out }}(\{q\}, n)\right] \prod_{m: \delta_{m} \leq \delta_{n}} p_{\text {out }}(\{q\}, m) \text {. }
$$

This equation can be better understand if we consider the scenario in Fig. 1. In this example, we have that the maximum expected advancement toward $t$ provided by a transmission from node $q$ is determined as a weighted sum of the geographical advancements provided by node $t$ and $n, w_{t} g_{q, t}+w_{n} g_{q, n}$, where the weighting parameter $w_{t}$ represents the probability that node $t$ correctly receive the message and $w_{n}$ represents the probability that only node $n$ correctly decode the message and thus it is selected as the next relay.

The $K$-one step look ahead ( $K$-OSLA) policy works as follows. At time slot $i$ (relay node set $a_{i}$ ), the transmitter closest to the destination $t$ is elected as the relay leader (denoted by $r^{*}$ ), i.e.,

$$
r^{*}=\underset{r \in a_{i}}{\operatorname{argmin}} \delta_{r}
$$

All nodes $n \in \mathcal{R}_{i}$ that correctly receive the message are ranked by node $r^{*}$ according to the overall expected advancement provided in the next two transmission slots, i.e.,

$$
\mu_{r^{*}, n}=g_{r^{*}, n}+g_{n} .
$$

Note that a high metric value is achieved by nodes $n$ providing both a good direct advancement toward $t$ (term $g_{r^{*}, n}$ ) and a good expected advancement $\left(g_{n}\right)$. The latter metric is particularly important to prevent the forwarding of the message toward connectivity holes, see [13]. Now, similar to $K$-Closest, node $r^{*}$ elects as relays the $K$ receivers with the highest value of $\mu_{r^{*}, n}$ and if less than $K$ nodes correctly decode the message, they are all elected as relays. Hence, nodes are ordered as in (3), where now (4) is replaced by

$$
\mu_{r^{*}, r_{i}^{(k)}} \geq \mu_{r^{*}, r_{i}^{(k+1)}}, \quad k=1,2, \ldots,\left|\mathcal{R}_{i}\right|-1,
$$

and set $a_{i+1}$ is provided by (5) .

A discussion is in order. The selection of the leader as the closest node to $t$ in the set $a_{i}$ provides a unique reference node for the calculation of the expected advancement toward $t$. In addition, this advancement is computed assuming a noncooperative transmission scheme where the leader is the only node that sends the message in the current time slot (term $\left.g_{r^{*}, n}\right)$, whereas the advancement provided in the next time slot by its neighbors that correctly receive the message is estimated using (7). This amounts to considering each neighbor $n$ as the only node that will be transmitting the message in the subsequent time slot $i+1$. As a matter of fact, the expected 
advancements computed in this way do not consider the effect of cooperative (and thus parallel) transmissions. Nevertheless, this reduces the computational complexity of the scheme, as a single node $r^{*}$ is used to represent an entire set $\left(a_{i}\right)$. Of course, suitable mechanisms for leader election and feedback collection must be also considered. However, these algorithms are outside the scope of this paper.

\section{C. $\eta$-dynamic One Step Look Ahead ( $\eta$-dOSLA)}

Both $K$-Closest and $K$-OSLA always select a fixed number of relays, potentially leading to an unnecessary waste of energy because also nodes that give a marginal contribution to the cooperative routing performance may be selected. In what follows, we extend the $K$-OSLA heuristic to take into account the impact of cooperation and let the number of relays be dynamic.

For the $\eta$-dynamic one step look ahead ( $\eta$-dOSLA) policy, we first define $\eta \in[0,1]$ as a parameter used to dynamically tune the number of cooperating nodes, as we will detail later. $\eta$-dOSLA uses the geographical advancement metric of (7) and, after set $\mathcal{R}_{i}$ has been ordered according to the metric $\mu_{r^{*}, n}$ of (9), the set $a_{i+1}$ is built iteratively as follows. Node $r^{*}$ starts by initializing the set $a_{i+1}$ so that it only contains the node with the highest rank

$$
a_{i+1}=\left\{r_{i}^{(1)}\right\}
$$

Then, $r^{*}$ calculates the expected advancement provided by the current set $a_{i+1}$ with respect to the highest rank node $r_{i}^{(1)}$ as

$$
\begin{aligned}
\gamma_{a_{i+1}}= & \sum_{n: \delta_{n} \leq \delta_{r_{i}^{(1)}}} g_{r_{i}^{(1)}, n} \\
& \times\left[1-p_{\text {out }}\left(a_{i+1}, n\right)\right] \prod_{m: \delta_{m} \leq \delta_{n}} p_{\text {out }}\left(a_{i+1}, m\right) .
\end{aligned}
$$

Comparing (12) with (7) we observe that, while in (7) the advancement is computed ignoring the cooperation of other nodes in $a_{i+1}, \gamma_{a_{i+1}}$ in (12) it includes the effects of the cooperation among all the nodes in the set $a_{i+1}$, which is gradually populated. Then, the normalized expected advancement is computed as

$$
\widetilde{g}_{a_{i+1}}=\frac{\gamma_{a_{i+1}}}{\delta_{r_{i}^{(1)}}},
$$

which represents the expected fraction of the distance $\delta_{r_{i}^{(1)}}$ covered by the cooperative transmission of the nodes in $a_{i+1}$.

If $\widetilde{g}_{a_{i+1}} \geq \eta$ the procedure terminates. Otherwise, node $r^{*}$ adds to set $a_{i+1}$ the next node in the ordered sequence $\mathcal{R}_{i}$ and recalculates the normalized expected advancement provided by all nodes in the new set $a_{i+1}$. At the generic iteration $v$, we have

$$
a_{i+1}=\left\{r_{i}^{(1)}, r_{i}^{(2)}, \ldots, r_{i}^{(v)}\right\}
$$

The iterative process is terminated either when $\widetilde{g}_{a_{i+1}} \geq \eta$ or when $v=\left|\mathcal{R}_{i}\right|$.

\section{Practical Considerations}

It can be noted that all the proposed algorithms are based on a distance metric between each node and the destination $t$. This is similar to the forwarding paradigm of geographical routing in wireless networks (see for example [14] and [15]), where it is assumed that each node is aware of its own position (e.g., exploiting GPS or some distributed localization service) and that the source is aware of the position of the destination. We make the same assumptions here, so that each node $n$ can determine its distance from the destination $\delta_{n}$ and exchange it with the other nodes of the network when needed. In this way, at each time slot $i$, we only require the knowledge of the local topology (i.e., $\mathcal{R}_{i}$ and $\delta_{r}, \forall r \in \mathcal{R}_{i}$ ) to determine the current relay set. Starting from these assumptions, the complexity of the proposed techniques varies and is represented by

- $K$-Closest: at each time slot $i$, the current transmitters order the set $\mathcal{R}_{i}$ according to (4), which has a complexity of $O(|\mathcal{N}| \log (|\mathcal{N}|))$;

- K-OSLA: each node needs to compute the maximum expected advancement (7), which has a complexity of $O\left(|\mathcal{N}|^{2}\right)$. Then, at each time slot $i$, the current relay leader $r^{*}$ orders the set $\mathcal{R}_{i}$ according to (10), which has a complexity of, at most, $O(|\mathcal{N}| \log (|\mathcal{N}|))$.

- $\eta$-dOSLA: each node needs to compute the maximum expected advancement (7), which has a complexity of $O\left(|\mathcal{N}|^{2}\right)$. At each time slot $i$, the current relay leader $r^{*}$ orders the set $\mathcal{R}_{i}$ according to (10), which has a complexity of $O(|\mathcal{N}| \log (|\mathcal{N}|))$. Moreover, $r^{*}$ iteratively builds the relay set $a_{i+1}$. At each iteration, node $r^{*}$ computes the expected advancement provided by the current relay set $a_{i+1}$ (i.e., (12)), which as a complexity of $O\left(|\mathcal{N}|^{2}\right)$. The maximum number of iterations required to compute the next relay set is $|\mathcal{N}|-2$ and thus, computing the next relay set has a complexity $O\left(|\mathcal{N}|^{3}\right)$.

Moreover, for a practical implementation of the proposed techniques, additional feedback mechanisms for packet decoding, relays selection and neighbor discovery must be designed. These mechanisms are out of scope for this work since our primary objective is investigating the effectiveness of different relay ranking criteria when used within an opportunistic routing protocol.

\section{NumERicAl RESUlts}

In this section we show numerical results of the network performance attained by the proposed algorithms. All the results of this section have been obtained using a $\mathrm{C}++$ simulator that assesses the performance of each algorithm using the Monte Carlo method. In the following graphs, we compare the delay and energy costs of the heuristic policies against the optimal policies obtained in [10]. In particular, we compare the performance of the distributed heuristic algorithms of Section III against the curves in [10, Fig. 5, page 515]. For the simulation scenario, we consider random networks with 21 nodes placed within a rectangular area of $50 \times d$ square meters as follows. Source and the destination node are placed in the 


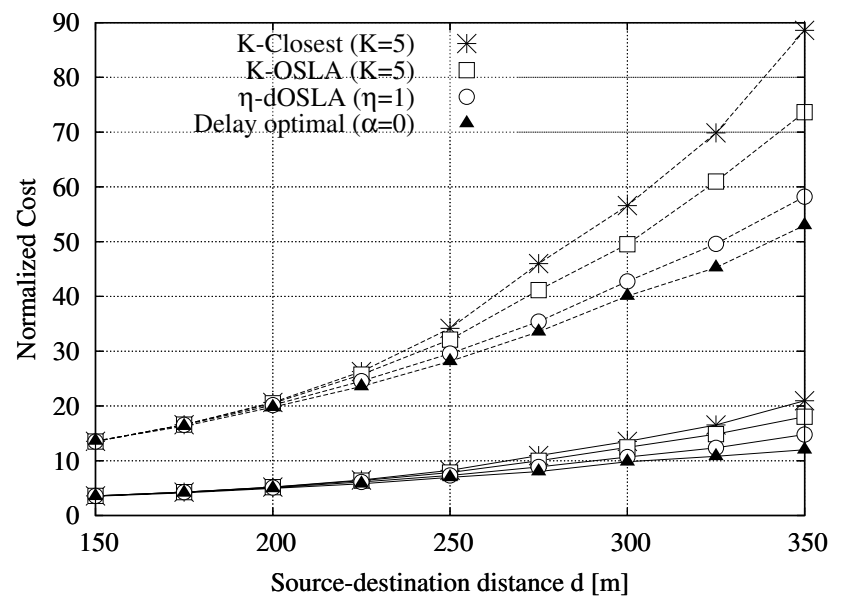

Fig. 2. Normalized energy and delay costs as a function of $d$ for optimal and heuristic policies, when the objective is delay minimization. Solid line: delay cost; dashed line: energy cost.

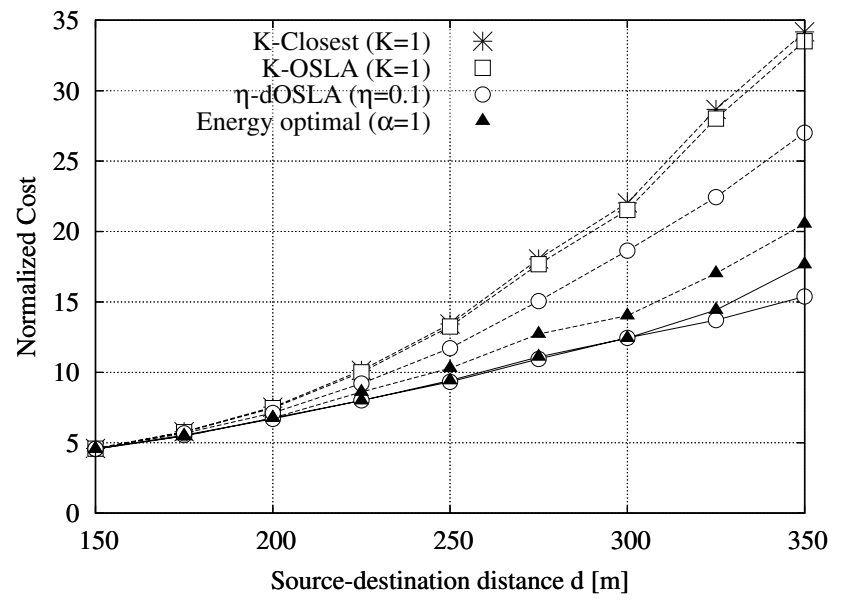

Fig. 3. Normalized energy and delay costs as a function of $d$ for optimal and heuristic policies, when the objective is energy minimization. Solid line: delay cost; dashed line: energy cost.

middle of the two opposite $50 \mathrm{~m}$ long sizes, therefore source and destination nodes are at a distance $d$. All the remaining nodes are randomly positioned within the area. Results in [10] are obtained when the maximum number of nodes that can cooperatively broadcast the message in a particular hop is set at $\chi_{\max }=5$. For comparison purposes, we therefore apply the same limitation to the heuristic policies proposed in Section III. This limitation implies $K \leq \chi_{\max }$ for policies $K$ Closest and $K$-OSLA, while for $\eta$-dOSLA we have to add the additional constraint that at most $\chi_{\max }$ nodes can cooperate at each hop. Note that this constraint implies that $\left|a_{i}\right| \leq \chi_{\max }$ and possibly leads to cases where $\widetilde{g}_{a_{i}}<\eta$, see (13). In all the results of this section the path loss exponent is $\kappa=3.5$. In addition, we set the data rate $R$ and the average signal to noise ratio in order to obtain, for a single active link, an outage probability of 0.2 at a distance of $30 \mathrm{~m}$.

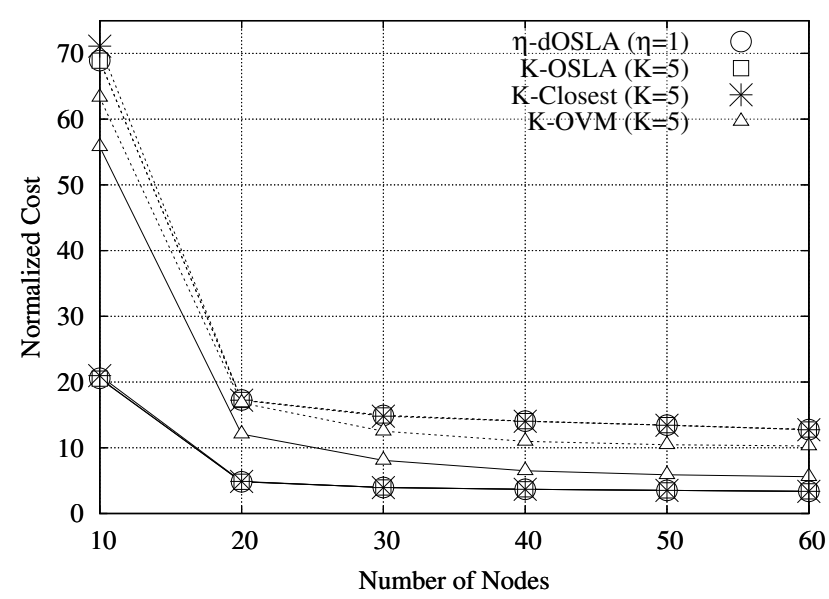

Fig. 4. Normalized energy and delay costs as a function of the number of nodes in the network for heuristic policies and OVM, when the objective is delay minimization. Solid line: delay cost; dashed line: energy cost.

Fig. 2 shows the energy and delay costs when the optimization objective is delay minimization. The curves in this figure have been obtained setting $\alpha=0$ in the cost function (2), $K=\chi_{\max }$ and $\eta=1$ and varying the distance $d$ between the source and the destination. We observe that for $d \leq 200 \mathrm{~m}$ all the schemes provide similar delay costs, while heuristic policies return a slightly higher energy consumption with respect to optimal policies. When $d$ increases, we see that $K$-Closest returns the worst performance in term of both energy and delay costs, while $K$-OSLA slightly outperforms $K$-Closest, and $\eta$-dOSLA still approaches the optimal performance with a delay increase of $22 \%$ and an energy increase of $10 \%$ in the worst case. Similar results are obtained in Fig. 3, where the optimization criterion is energy minimization. In this figure we set $\alpha=1, K=1$ and $\eta=0.1$. Note that setting $K=1$ implies that no cooperation is allowed in both $K$-Closest and $K$-OSLA (and thus energy and delay costs coincide, see (2)), while $\eta>0$ allows the simultaneous transmission from different nodes. As before, $\eta$-dOSLA outperforms $K$-Closest and $K$-OSLA and, despite attaining higher energy expenditure, achieves better delay performance with respect to the optimal policies (note that this is allowed because the optimization criterion of the optimal policies is energy minimization).

In addition, we compared the performance of the proposed heuristic policies with that of the opportunistic virtual MISO (OVM) protocol proposed in [12]. OVM considers that, at each hop, the current transmitter can be assisted by one relay. Since in our heuristic policies we can tune the number of cooperating nodes, we extended OVM in a similar way. We call this implementation $K$-OVM, where $K$ represents the maximum number of nodes that can cooperatively forward the message to the next hop. Here we consider the same network structure of the previous figures, except for the size of the rectangular area, which is now $150 \times 150$ square meters.

Fig. 4 shows the energy and delay costs as a function of the number of nodes in the network when the objective is delay 


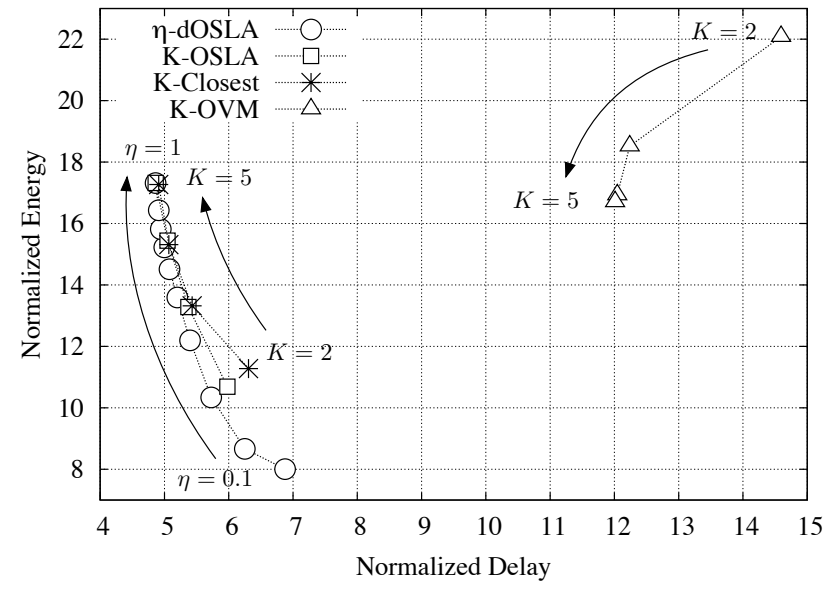

Fig. 5. Energy as a function of the delay for heuristic policies and OVM. The curves are obtained for 20 nodes, varying $\eta \in(0,1]$ and $K \in\{2,3,4,5\}$.

minimization. In this figure, the curves have been obtained setting $K=5$ and $\eta=1$. We see that our schemes outperform OVM in all the considered scenarios. In addition, we observe that $K$-OSLA and $\eta$-dOSLA perform slightly better than $K$ Closest when the number of nodes is less than 30, while all the three schemes perform closely for higher node densities. This is reasonable since the additional information about the expected advancement exploited by $K$-OSLA and $\eta$-dOSLA is meant to prevent the forwarding of the message toward connectivity holes, that are more frequent for sparser networks.

In Fig. 5, we set the number of nodes to 20 and we obtained the points by varying $\eta$ in $(0,1]$ for $\eta$-dOSLA and $K$ in $\{2,3,4,5\}$ for $K$-Closest, $K$-OSLA and $K$-OVM. We observe that our schemes outperform $K$-OVM in terms of delay cost for all the values of $K$ and $\eta$. As expected, $K$-OSLA improves over $K$-Closest in terms of both energy and delay, especially for small values of $K$ ( $K \leq 3$ in the figure), while for $K \in\{4,5\}$ it provides a small delay improvement at the expense of a slight increase in energy expenditure. In addition, we observe that $\eta$-dOSLA outperforms all the other schemes and allows for a more refined tuning between energy and delay. Finally, it is interesting to notice that while in our schemes increasing the number of cooperating nodes leads to a decrease in the delay experienced by the message and a consequent increase in the energy consumption, in $K$-OVM increasing $K$ has the effect of simultaneously decreasing both energy and delay and this reflects the different working principles of the two schemes.

\section{Conclusions}

In this paper, three algorithms for the selection of cooperating nodes in multihop wireless networks implementing a virtual MISO transmission have been proposed. The aim of these policies is the minimization of a cost obtained as a linear combination of delay and energy consumption. The three policies allow the selection of the cooperating nodes at a local level among the nodes that receive the message at each hop, thus being viable for a practical implementation. They differ for various look-ahead strategies that realize a locally greedy approach for the solution of the otherwise complex global optimization problem. In a performance comparison with the optimal centralized approach, the heuristics exhibit very limited losses and in any case outperform approaches that had been presented in the literature, thus being of interest for their use in future networks.

\section{REFERENCES}

[1] T. Cover and A. Gamal, "Capacity theorems for the relay channel," IEEE Trans. on Info. Theory, vol. 25, no. 5, pp. 572-584, Sept. 1979.

[2] A. Bletsas, A. Khisti, D. P. Reed, and A. Lippman, "A simple cooperative diversity method based on network path selection," IEEE Jour. on Selec. Areas in Commun., vol. 24, no. 3, pp. 659-672, Mar. 2006.

[3] A. E. Khandani, J. Abounadi, E. Modiano, and L. Zheng, "Cooperative routing in static wireless networks," IEEE Trans. on Commun., vol. 55, no. 11 , pp. 2185-2192, Nov. 2007.

[4] A. Ibrahim, Z. Han, and K. Liu, "Distributed energy-efficient cooperative routing in wireless networks," IEEE Trans. on Wireless Commun., vol. 7, no. 10, pp. 3930-3941, Oct. 2008.

[5] M. Dehghan, M. Ghaderi, and D. L. Goeckel, "Cooperative diversity routing in wireless networks," in Proc. WiOpt, Avignon, France, May 2010.

[6] S. Lakshmanan and R. Sivakumar, "Diversity routing for multi-hop wireless networks with cooperative transmissions," in Proc. IEEE SECON, Rome, Italy, June 2009

[7] J. E. Wieselthier, G. D. Nguyen, and A. Ephremides, "Energy-efficient broadcast and multicast trees in wireless networks," Mobile Networks and Applications, vol. 7, pp. 481-492, Dec. 2002.

[8] C. Lott and D. Teneketzis, "Stochastic routing in ad-hoc networks," IEEE Trans. on Automatic Control, vol. 51, no. 1, pp. 52-70, Jan. 2006.

[9] M. Naghshvar, H. Zhuang, and T. Javidi, "A general class of throughput optimal routing policies in multi-hop wireless networks," in Proc. 47th Allerton conf. on commun., control, and computing, Monticello, Illinois, USA, Apr. 2009.

[10] M. Rossi, C. Tapparello, and S. Tomasin, "On optimal cooperator selection policies for multi-hop ad hoc networks," IEEE Trans. Wireless Commun., vol. 10, no. 2, pp. 506-518, Feb. 2011.

[11] G. Jakllari, S. V. Krishnamurthy, M. Faloutsos, P. V. Krishnamurthy, and O. Ercetin, "A cross-layer framework for exploiting virtual MISO links in mobile ad hoc networks," IEEE Trans. on Mobile Computing, vol. 6, no. 6, pp. 579-594, June 2007.

[12] V. Nguyen and D. Perkins, "An opportunistic virtual MISO (OVM) protocol for multi-hop wireless networks," in Proc. 5th IEEE Int. Symp. on Wireless Pervasive Computing (ISWPC), Modena, Italy, May 2010.

[13] N. Ahmed, S. S. Kanhere, and S. Jha, "The holes problem in wireless sensor networks: a survey," SIGMOBILE Mob. Comput. Commun. Rev., vol. 9, pp. 4-18, Apr. 2005.

[14] I. Stojmenovic, "Position-based routing in ad hoc networks," Соттиnications Magazine, IEEE, vol. 40, no. 7, pp. 128 -134, jul 2002.

[15] R. Jain, A. Puri, and R. Sengupta, "Geographical routing using partial information for wireless ad hoc networks," Personal Communications, IEEE, vol. 8, no. 1, pp. 48 -57, feb 2001. 